\title{
Peri-abortion contraceptive care: Can we reduce the incidence of repeat abortions?
}

\author{
Malini Garg, MB BS, DGO, DFFP, Senior House Officer; Madan Singh, MB ChB, FRCOG, DM, MFFP Emeritus Consultant in \\ Gynaecology, Royal Victoria Infirmary, Newcastle Upon Tyne, UK. \\ Diana Mansour, BM BCh, MRCOG, MFFP, Consultant in Community Gynaecology and Reproductive Healthcare \\ Newcastle General Hospital, Newcastle Upon Tyne, UK.
}

\begin{abstract}
Correspondence: Mr M Singh, Department of Gynaecology, Royal Victoria Infirmary, Queen Victoria Road, Newcastle Upon Tyne, NE1 4LP, UK
\end{abstract}

Tel: 0191232 5131, Fax: 01912275173

(Accepted June 26 ${ }^{\text {th }}, 2000$ )

\begin{abstract}
Summary
Introduction. It is of great importance for repeat unwanted pregnancies to be prevented rather than aborted. We therefore sought to: determine the reasons for contraceptive failure in women seeking repeat abortions; audit the periabortion contraception services offered at our hospital, and make recommendations regarding peri-abortion contraception services based on the above findings.

Method. A self-administered questionnaire was used to determine the contraceptive practices and details of periabortion contraceptive counselling received by 50 women undergoing a repeat, and 83 women undergoing a firsttime, abortion.

Results. Ninety-eight percent of women undergoing a repeat abortion reported using contraception at the time of conception, as compared to 83\% of women undergoing a first-time abortion. This difference was significant $(p=0.009)$. Condoms were the main method used by $57 \%$ of women undergoing a repeat and $70 \%$ of women undergoing a first-time abortion. The oral contraceptive pill (OCP), including both combined oral contraceptive and progestogen-only pill, was the main method used by $37 \%$ of women undergoing a repeat and $25 \%$ undergoing a first-time abortion. Both these methods were found to be ineffective because of user-dependent failures. All women received periabortion contraceptive counselling, but the perceived contents varied. Follow-up contraceptive appointments were made in less than half of women. Although most women chose an optimal contraceptive method as a result of the counselling, compliance with the chosen method in women undergoing repeat abortions was poor.

Conclusions. Standards of audit were met with regards to receipt of contraceptive counselling and agreeing a contraceptive method before discharge. The content of this counselling needs to be improved. The ineffectiveness of the OCP and barrier methods of contraception needs to be highlighted during counselling. Adequate follow-up arrangements need to be provided to ensure compliance of the chosen method of contraception.
\end{abstract}

\section{Key words}

contraception, counselling, repeat abortions

\section{Introduction}

Apart from moral and ethical considerations, induced abortions carry a significant psychological and physical morbidity. It is therefore of great importance for repeat unwanted pregnancies to be prevented rather than aborted. Guidelines for Induced Abortions issued by the Royal College Of Obstetricians and Gynaecologists ${ }^{1}$ and endorsed by the National Health Service Executive emphasise the importance

\section{Key message points}

- There is a significant, but preventable, incidence of repeat abortions.

- Thorough peri-abortion contraceptive counselling and a structured follow-up strategy are essential to ensure patient acceptance and effective contraception continuation.

- Women need to be counselled that OCPs require user compliance and that barrier methods used alone are ineffective.

- The superior effectiveness of the IUD and progestogen injectable methods when compared to the OCPs and barrier methods needs to be highlighted during this counselling.

of peri-abortion contraception counselling. In fact, studies have shown that women seeking to avoid an unwanted pregnancy are more likely to use contraception if they have suffered an abortion in the past. ${ }^{2}$ Despite this, there seems to be a significant incidence of repeat abortions. ${ }^{3}$ Our aims were three-fold: to determine the reasons for contraceptive failure in women seeking repeat abortions; to audit the peri-abortion contraception services offered at our hospital, and to make recommendations regarding peri-abortion contraception services based on the above findings. To our knowledge, this is the first study from the UK which has specifically studied women undergoing repeat abortions in this way.

\section{Method}

Standards of care for purposes of auditing peri-abortion contraception services

These were as described in the recent new Guidelines for Induced Abortion document of the Royal College Of Obstetrics and Gynaecologists ${ }^{1}$ and current literature. ${ }^{4}$ They are as follows:

1. All patients should receive information on available methods of contraception and health hazards of abortions.

2. A plan of contraception usage should be agreed upon before discharge for all patients.

3. All patients should be followed-up at 2 weeks by the family doctor or gynaecologist to sort out any problems with contraception usage.

Details regarding the contraceptive practice of these women, perceived reasons for failure of contraception, use of emergency contraception (EC) and details of periabortion contraception counselling were determined using a self-administered questionnaire (available from the authors). Women having had a previous abortion were also asked whether they had undergone any follow-up after their previous abortion. This was administered by the nurse looking after the patient during routine clerking and admission on the day of their abortion. Demographic characteristics, details of contraceptive method chosen as a result of counselling, and any follow-up arrangements were 
determined from the case-records of these patients.

Consent for the study was obtained from the women at the time of administering the questionnaires. A pilot study with 15 patients undergoing a first-time, and 15 undergoing a repeat, abortion was performed to determine the validity of the questionnaire and feasibility of the study. The questionnaire was suitably modified. Fifty consecutive women undergoing a repeat and 83 women undergoing a first-time abortion were studied.

Data from the forms were entered onto a database by our audit facilitator. Epi info software was used to construct the database. The Mann-Whitney $U$ test for non-parametric quantitative data was used to test for difference in the ages of women undergoing a repeat or first-time abortion. The Chi-square test was used to test for differences in contraceptive use between these two groups. The McNemar's test for matched nominal variables was used to test the difference between the contraceptive method chosen at the termination of pregnancy (TOP) clinic and the actual method used after discharge. A value of $p<0.01$ was considered to be significant. Statistical calculations were performed using Minitab software.

\section{Results}

Eighty-three women undergoing a first-time and 50 women undergoing a repeat abortion completed the questionnaires. The median age of women undergoing a first-time abortion was 22 (range: 15-40) years. The median age of women undergoing a repeat abortion was 26 (range: 17-42) years. There was a statistically significant difference in the ages of these two groups of women $(p=0.0006)$. Forty-four of the 50 women undergoing a repeat abortion had had one previous abortion, five had had two previous abortions and one had had four previous abortions.

Ninety-eight percent of women undergoing a repeat abortion reported using contraception at the time of conception, as compared to $83 \%$ of women undergoing a firsttime abortion. This difference was significant $(\mathrm{p}=0.009)$. Condom was the prime method used by $57 \%$ of women undergoing a repeat, and $70 \%$ of women undergoing a firsttime, abortion $(\mathrm{p}=0.83)$. The OCP was the main method used by $37 \%$ of women undergoing a repeat, and $25 \%$ undergoing a first-time, abortion $(\mathrm{p}=0.049)$. Details of other methods used and the reasons for failure are shown in Table 1.

Twelve percent of women undergoing a repeat and $10 \%$ undergoing a first-time abortion reported use of EC. Reasons for non-usage of EC are shown in Table 2. There was no significant difference between these two groups with regards to use of EC $(\mathrm{p}=0.588)$.

Table 2 Emergency contraception in women undergoing a first-time or repeat abortion. Figures in parentheses are percentages.

\begin{tabular}{llll} 
& Women & Women & $\mathrm{p}$ \\
& undergoing & undergoing & Value* \\
a repeat & a first-time & \\
abortion & abortion \\
& $(\mathrm{n}=50)$ & $(\mathrm{n}=83)$ & \\
\hline
\end{tabular}

Recognised possibility

of pregnancy or

contraceptive failure

- Did not use EC

- Did not know about EC

- Could not obtain EC

- Did not know where to obtain EC

- Did not think about EC at the time

Did not think that pregnancy would

occur or contraceptive method

would fail

$\begin{array}{rr}33(66) & 60(72) \\ 29(88) & 54(90) \\ 6(21) & 7(13) \\ 2(7) & 3(5) \\ 1(3) & 1(2) \\ 20(69) & 43(79)\end{array}$

0.588

(13)

$3(79)$

$17(34) \quad 23(28)$

* p value obtained using the Chi-square test.

Ninety-eight percent of women undergoing a first-time or repeat abortion received peri-abortion contraceptive advice. Details of the advice received are shown in Table 3. A contraceptive method was chosen by all women before discharge. Condoms were chosen by $6 \%$, the OCP by $61 \%$, progestogen-only injection by $16 \%$, and the intrauterine device (IUD) by $14 \%$ of women undergoing a first-time abortion. In women undergoing a repeat abortion, $17 \%$ had chosen the condom method after their previous abortion, but $57 \%$ were using this method at the time of this conception. Twenty-two percent of these women had chosen a progestogen injection (Depo Provera) method, but only $2 \%$ were using it at the time of

Table 1 Contraception usage and reasons for contraceptive failure in women undergoing a first-time or repeat abortion. Figures in parentheses are in percentages.

\begin{tabular}{|c|c|c|c|}
\hline $\begin{array}{l}\text { Contraceptive method and } \\
\text { reasons for failure }\end{array}$ & $\begin{array}{l}\text { Women undergoing a } \\
\text { repeat abortion }(n=50)\end{array}$ & $\begin{array}{l}\text { Women undergoing a } \\
\text { first-time abortion }(n=83)\end{array}$ & p Value* \\
\hline No contraception & $1(2)$ & $14(17)$ & 0.009 \\
\hline Condoms & $28(57)$ & $48(70)$ & 0.836 \\
\hline - split condom & 13 & 16 & \\
\hline - did not use all the time & 5 & 11 & \\
\hline - condom came off & 1 & 8 & \\
\hline - unexplained failure & 9 & 13 & \\
\hline Oral contraceptive pill & $18(37)$ & $17(25)$ & 0.049 \\
\hline - supply of pills ran out & 5 & 2 & \\
\hline - forgot to take pill & 7 & 9 & \\
\hline - had vomiting and diarrhoea & 2 & 4 & \\
\hline - drug interactions & 1 & 0 & \\
\hline - unexplained failure & 3 & 2 & \\
\hline Progestogen injections (Depo Provera) & $1(2)$ & 0 & \\
\hline - unexplained failure & 1 & 0 & \\
\hline IUD & $2(4)$ & $1(1)$ & \\
\hline - removed before other surgery & 0 & 1 & \\
\hline - taken out to go onto pill & 1 & 0 & \\
\hline - unexplained failure & 1 & 0 & \\
\hline Female sterilisation & 0 & 0 & \\
\hline Male sterilisation & 0 & $1(1)$ & \\
\hline Rhythm method & 1 & 1 & \\
\hline
\end{tabular}

* p value obtained using the Chi-square test 
Table 3 Peri-abortion contraceptive advice received and its contents. Figures in parentheses are percentages.

\begin{tabular}{|c|c|c|}
\hline & $\begin{array}{l}\text { Women } \\
\text { undergoing a } \\
\text { repeat abortion } \\
(\mathrm{n}=50)\end{array}$ & $\begin{array}{l}\text { Women } \\
\text { undergoing a } \\
\text { first-time abortion } \\
(\mathrm{n}=83)\end{array}$ \\
\hline Received advice at TOP clinic & $49(98)$ & $81(98)$ \\
\hline Barrier methods are unreliable & $20(40)$ & $30(36)$ \\
\hline $\begin{array}{l}\text { Preferred options are pills/ } \\
\text { injections/IUD/sterilisation }\end{array}$ & $38(76)$ & $60(72)$ \\
\hline Options discussed with relevance & 21 & $36(44)$ \\
\hline Information about emergency & & \\
\hline contraception & $13(26)$ & $34(41)$ \\
\hline immediately after TOP & $38(76)$ & $59(71)$ \\
\hline Health hazards of abortions & $27(54)$ & $34(41)$ \\
\hline
\end{tabular}

this conception. A comparison of the contraceptive method chosen at the TOP clinic and the method being used in women undergoing a repeat abortion is shown in Figure 1. There was a significant increase in the reported use of the condom method after discharge $(p<0.001)$ in this group of women.

There were no follow-up arrangements for $51 \%$ of women undergoing a repeat, and $57 \%$ undergoing a firsttime, abortion. Follow-up had been arranged with the family planning clinic in $30 \%$ of all women, and with the general practitioner in the remaining women.

\section{Standards of audit}

Standard 1. All women received peri-abortion contraceptive advice. This advice was varied and was inadequate in about $50 \%$ of the cases.

Standard 2. A contraceptive plan was agreed for every woman before discharge.

Standard 3. The standards for follow-up in every patient was met in about $50 \%$ of cases.

\section{Discussion}

Repeat abortions represent a significant proportion of abortions world-wide. Studies from the United States ${ }^{2}$ and Europe $^{5}$ reveal an incidence ranging between $20-30 \%$. A recent study from the UK revealed an incidence of $27 \% .^{6}$ One of the studies has shown that the incidence of repeat abortions has been rising over the last few years. ${ }^{2}$ It is thus very important to study the factors responsible for contraceptive failure in this group of women in an effort to remedy the situation.

Our study found that the reported use of contraception was significantly higher in women undergoing repeat abortions than in those undergoing a first-time abortion (98\% vs. 83\%; $p=0.009$ ). This is similar to studies from the United States, ${ }^{2}$ although other groups have found no difference. ${ }^{7}$ The actual rate of contraceptive use may be lower than our findings; women having abortions may not admit to not using a contraceptive method. In our study, women undergoing a repeat abortion reported using more ineffective forms of contraception. The most common form of contraception reported to be used by women seeking repeat abortions was the condom $(57 \%)$, followed by the OCPs $(37 \%)$. Although there was a slight shift to an increased use of the OCPs amongst women seeking repeat abortions, the reported use of different contraceptive methods was not significantly different from those undergoing a first-time abortion (Table 1).

The promotion of barrier methods of contraception as protection from HIV has increased the frequency with

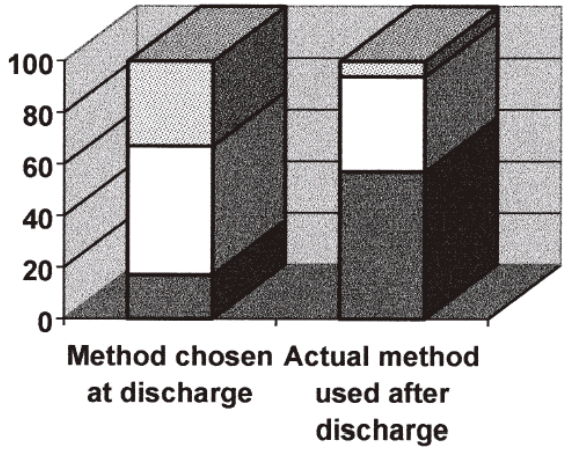

Others 口OCPs Condoms

Figure 1 A comparison of contraceptive methods chosen as a result of counselling and actual use in women undergoing a repeat abortion. This shows a significant increase in the use of condoms after discharge $(p<0.001 ;$ McNemar's test $)$.

which unplanned pregnancies are associated with condom use. ${ }^{6}$ Although both the condom and OCP methods have a low pearl index, ${ }^{8}$ they have much higher failure rates in practice. These are mainly due to user error and are associated with improper use or 'accidents' in the case of condoms, and missed pills in the case of OCPs. ${ }^{6}$ Our study showed similar problems with these contraceptive methods in women undergoing repeat abortions (Table 1). Such user errors, together with side-effects of nausea and vomiting associated with OCPs, accounted for $68 \%$ of all the repeat abortions. Thus, use of alternative forms of contraception, such as the IUD and progestogen injections, would have prevented $68 \%$ of repeat abortions in our series. This higher effectiveness of the IUD and progestogen injection methods of contraceptives needs to be highlighted during periabortion contraceptive counselling.

There was one failure from the use of the IUD because of removal before surgery, and another because the IUD was removed to change over to the OCP. These could have been prevented by asking about sexual intercourse before removal of an IUD, and not removing an IUD until alternative contraceptive methods have been established. These are important points that are easily overlooked and could reduce the effectiveness of the IUD.

The use of EC was very poor. There was no significant difference in the reported use of EC between women undergoing a first-time or repeat abortion (Table 2). Some earlier studies have suggested that this is due to a lack of awareness of EC. ${ }^{9,10}$ However, our study showed that the poor uptake did not relate to a lack of awareness of contraception, but was mainly due to lack of motivation to use EC. It is thus important that this is taken into account by the various agencies who are undertaking education campaigns to improve awareness of EC.

Advice on peri-abortion contraception was given to all patients in our study. The content of this advice varied (Table 3). Although most women were told that the preferred options of contraception were the OCPs, IUD or progestogen-only injection forms of contraception, only half of these women were told about the ineffectiveness of barrier contraception. A discussion of the various contraceptive options in relation to individual circumstances was made in $40 \%$ of the women. Less than half were told of the option of using EC if their primary method failed.

We studied the choice of contraception made as a result of counselling at the TOP clinic, and related this to the reported use of the contraceptive method in women undergoing a repeat abortion. The majority of the initial abortions and subsequent counselling had been carried out 
at in our unit. Although initially most of these women chose a non-barrier method of contraception, when seen for their repeat abortion, they reported using the male condom as their contraceptive method. This change in contraceptive method was highly significant $(\mathrm{p}<0.001)$. This may be in part due to the fact that although peri-abortion contraceptive counselling mentioned non-barrier forms of contraception as the preferred methods, it failed to highlight the ineffectiveness of barrier methods. Moreover, most of the women had no follow-up. A follow-up visit with their GP or family planning clinic would have allowed for a reemphasis of the effectiveness of the non-barrier methods, and would ensure patient acceptance and improved continuation of the contraceptive method chosen. Other groups from Sweden ${ }^{5}$ and Denmark ${ }^{7}$ in Europe, and from the United States, ${ }^{2}$ who have studied contraceptive failure in women undergoing a repeat abortion, emphasise the need for detailed counselling and effective follow-up of these women. The Birth Control Trust ${ }^{11}$ advocate early follow-up as a routine for all women following abortion.

Our study cannot give any data on women who having had an abortion, do not subsequently have a repeat abortion. The results of the study may be biased because women having a repeat abortion may comprise only a small proportion of women who have had an abortion. Thus, it may be that a large number of women who have had an abortion have been successful in their contraceptive practices. Nevertheless, our study yields useful information on the contraceptive practices and highlights the importance of detailed contraceptive counselling in this specific subgroup of British women.

Our study confirms that women undergoing repeat abortions are highly motivated users of contraception. They make optimum choices when given appropriate counselling, but do not comply with this choice. Thus, it is likely that a more thorough peri-abortion contraceptive counselling with better follow-up would facilitate compliance and result in more effective use of contraceptive methods. This is strongly supported by the recent new guidelines on abortion from the Royal College of Obstetrics and Gynaecology. Such a strategy should translate into a reduced incidence of repeat abortions.

\section{Conclusion}

Various changes in our current peri-abortion contraceptive care arrangements could have a favourable impact on the incidence of repeat abortions, as follows:

- more thorough peri-abortion contraceptive counselling

- emphasis that OCPs require user compliance and that barrier methods used alone are ineffective

- highlighting the superior effectiveness of the IUD and progestogen injectable methods when compared to the OCPs and barrier methods during this counselling

- a structured follow-up strategy to ensure patient acceptance and improve effective contraceptive continuation.

We are in the process of incorporating these changes at our institute. We plan to re-audit the peri-abortion contraceptive care arrangements in one year's time.

\section{Acknowledgements}

We are grateful to Caroline Stokoe for the assistance rendered with regard to the compilation of the database. This study would not have been possible without the co-operation of the women undergoing abortions at our hospital. We are also grateful to Sister C Hudson and her dedicated nursing staff who ensured that the women received and completed the questionnaires.

\section{Statements on funding and competing interests}

Funding. None.

Competing interests. None.

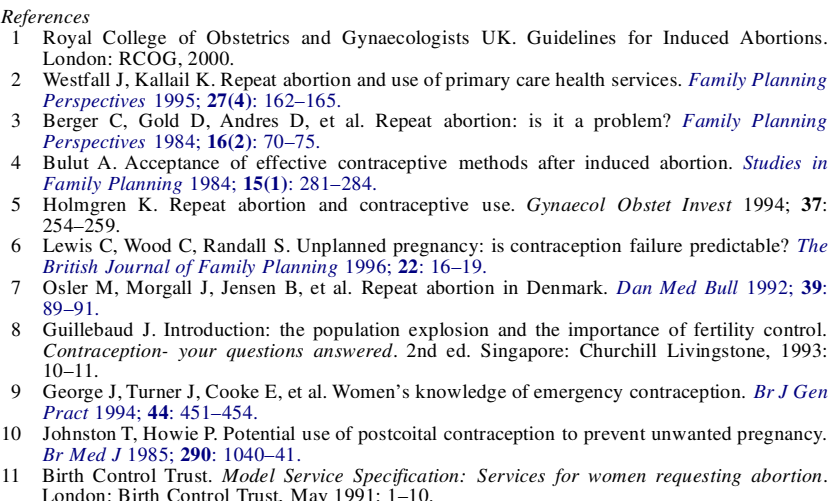

Guillebaud J. Introduction: the population explosion and the importance of fertility control. Contraception- your questions answered. 2nd ed. Singapore: Churchill Livingstone, 1993: $10-11$

George J, Turner J, Cooke E, et al. Women's knowledge of emergency contraception. Br J Gen Pract 1994; 44: 451-454

Johnston T, Howie P. Potential use of postcoital contraception to prevent unwanted pregnancy. Br Med $1985,290.1040-41$. Birth Control Trust. Model Service Specification: Services for women requesting abortion. 